\title{
PERIARTERITIC LESIONS OF THE CAROTID SIPHON WITH THE CLINICAL FEATURES OF A CAROTID INFRACLINOIDAL ANEURYSM
}

BY

\section{EDUARDO TOLOSA}

\section{From the Neurosurgical Service of the Neurological Institute, Barcelona, Spain}

The clinical diagnosis of subclinoid carotid aneurysms is generally based on the characteristic association of two features: (a) pain in the first division of the trigeminal nerve ; and $(b)$ progressive paralysis, partial or total, of the oculomotor nerve and occasionally of the fourth, sixth, and the fifth cranial nerves. Although the symptomatic association just described is highly characteristic, it is only through the use of cerebral angiography that we are able to establish the diagnosis with certainty.

In some cases of the syndrome, arteriography may indicate the presence of a tumour in the base of the skull (the carotid siphon running a zigzag course or showing an abnormal opening of its second curve, etc.), but in others there may be a non-aneurysmal vascular process of the intracranial carotid. In this paper I wish to deal with the latter possibility, and to report a case in which the evolution and clinical condition of the patient seemed to point to the diagnosis of intracavernous carotid aneurysm, while the arteriographic examination suggested the existence of disease of the wall of the carotid siphon, and this was subsequently confirmed at necropsy.

\section{Case Report}

S. M. M., a man aged 47 , was admitted to the Neurological Service of the Neurological Institute in September, 1949. His present illness began three years before, the initial symptom being pain in the left orbital region. The ache was slight but continuous for a few days. A month before the patient's admission pain was again experienced in the territory of the first branch of the left trigeminus : it became violent and continuous, and was accompanied by vomiting. During the past eight days the patient had noticed ptosis of the upper left eyelid which increased rapidly into a total left ophthalmoplegia.

Funduscopic examination revealed no important pathological changes. The visual fields were full. Visual acuity : right eye, 1 ; left eye, 0.5 .
The third, fourth, and sixth cranial nerves on the left side were completely paralysed. The left corneal reflex was weak. Objective sensibility was normal in the region of the left fifth nerve. No disturbances of the trigeminal motor functions or defects in the remaining cranial nerves were found. The neurological examination of the trunk and limbs was also negative. Blood pressure was $140 / 70 \mathrm{~mm}$. $\mathrm{Hg}$.

Wassermann's test in the blood was negative.

The radiological examination of the skull showed only an increase in density of the small sphenoidal wing on the left side, the upper limit of which appeared ill-defined.

In the angiographic pictures, the anterior and the middle cerebral arteries appeared in their normal positions but the carotid siphon showed in the lateral view (Fig. 1) a segmentary narrowing at the level of portion $\mathbf{C}_{2}$.

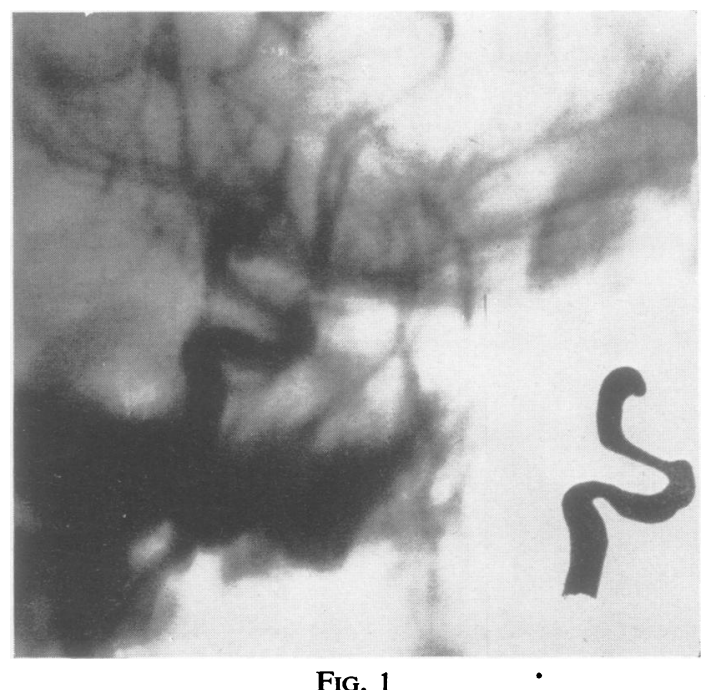

This examination apparently excluded the diagnosis of saccular aneurysm or of meningioma. The existence of a malignant tumour in the base of the skull seemed 
improbable since in these cases the carotid siphon ordinarily shows other changes. Although the arteriographic findings pointed to a local vascular lesion (arteritis, arteriosclerosis) this diagnosis was somewhat difficult to reconcile with the result of the neurological and radiological examinations. It was decided therefore to explore surgically the region of the sella turcica.

On September 29, 1949, under general intratracheal anaesthesia, a left transfrontal intradural exploration of the region of the optic chiasm was made. The optic nerves were short. No lesion was found either in the parasellar region, in which only slight arachnoidal opacity was discovered, or in the sphenoidal ridge. The temporal pole, the anterior part of the Sylvian fissure, and the posterior side of the great sphenoid wing were explored with negative results. The tension was still raised and suggested the possible existence of a deep tumour. However, the dural incisions and the bone and cutaneous flaps were closed.

The post-operative condition was satisfactory on the first and second days, but on the third day the patient fell into a torpor and thence into a coma, dying on October 2, 1949.

At the post-mortem examination no aneurysm was seen in the arterial circle of Willis and no tumour was disclosed in the brain or at the base of the skull. The latter presented an asymmetric shape, the sphenoid ridge and the superior edge of the petrous pyramid on the

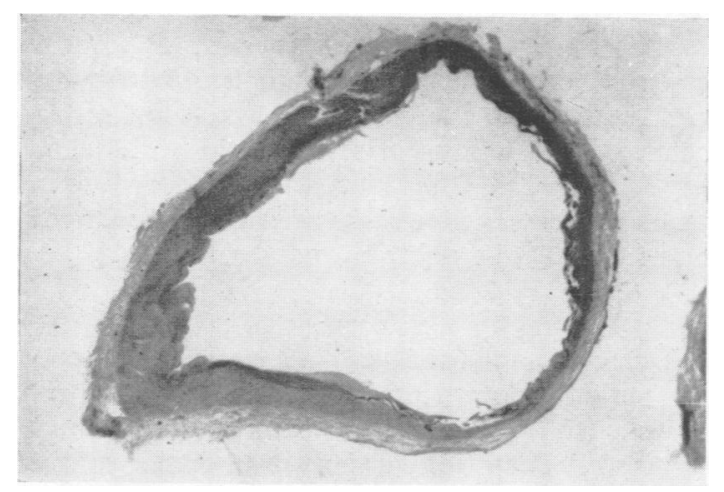

FIG. 2a

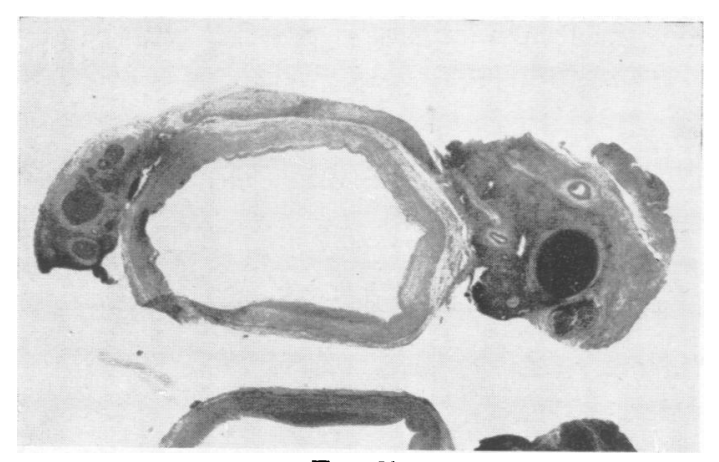

Fig. 2b left side being on a more dorsal position than those on the right side. The cavernous sinuses were opened on both sides. On the left the intracavernous portion of the carotid was wrapped in granulomatous tissue which, however, did not completely obstruct the lumen of the sinus. This segment of the left carotid was also markedly narrowed. Altogether the curvatures of the left carotid siphon were wider than those of the right side, and its walls were excessively rigid and thick, and of a yellowish colour which contrasted with the whitish-grey of the right one.

Serial sections were made of the carotid siphons in order to compare their microscopical appearance. The left intracavernous carotid (Fig. 2b) in some parts showed a thickening of the adventitia. Around it there was a cuff of non-specific granulation tissue including the adjoining nervous trunks. No endoarteritic or mesoarteric lesions were found. The right intracavernous carotid (Fig. 2a) was practically normal in appearance.

Sections were made from various regions of the hemispheres, no pathological features being observed in either the nervous tissues or in the vessels. There was obvious oedema of the cerebral tissue in the white matter of the left frontal lobe.

\section{Comment}

This report shows that periarteritic lesions of the intracavernous section of the carotid artery may bring about a clinical syndrome resembling that of an aneurysm of the infraclinoid carotid. The classic paper by Jefferson (1938) shows that the syndrome observed in this case is almost always caused by aneurysms of the anterior portion of the intracavernous carotid. This syndrome is in contrast to that of the aneurysms of the posterior third in which pressure directly on the trigeminal root affects the whole of the trigeminal sensitive territory (sometimes including the motor branch) and to that of aneurysms of the middle third where the first and second branches of the fifth nerve are reached.

Discussing the diagnosis of these aneurysms, Jefferson remarked that the cavernous syndrome may be found in connexion with syphilis, with malignant tumours at the base of the skull, with a parasellar meningioma, and also, although exceptionally, with a hypophyseal adenoma, but no allusion is made to the possibility of lesions such as those just described. The association of symptoms of the pericarotid nerves to nonaneurysmal lesions of the carotid siphon is not, however, as exceptional as might be believed.

In a case of a 63-year-old patient recently observed by me, who presented with a right hemiplegia, pains in the left half of the head, and paralysis of the left third nerve, the carotid angiography (left side) showed that not only did the anterior cerebral artery not fill, but there were irregularities in the 
calibre of the siphon with many segments appreciably narrowed (Fig. 3). List and Hodges (1946) have described a case with a narrowing of the right carotid siphon (probably associated with an occlusion of the ophthalmic artery) in which a transient partial oculomotor paralysis of the right side was present. Finally, the participation of the third homolateral nerve has been noted in some cases of thrombosis of the carotid.

The pathogenesis of the oculomotor palsy of this case must be due to the inclusion of the pericarotid nerves in the inflammatory process which enveloped the carotid siphon. The carotid lumen was perfectly patent, and the lesions, being exclusively pericarotid, make the case quite different from those of carotid thrombosis.

The changes in the intima of the vascular lesion lead to the question of whether the syndrome could be related to the disease of Winiwater-Buerger. However, in the literature on Buerger's cerebral disease (Krayenbühl, 1945 ; Davis and Perret, 1947) lesions are not described which are comparable to those in this case, in which the arterial lumen was completely free of thrombosis and endoarteritic lesions were entirely lacking.

It would also be possible to classify this case as one of thrombosis of the cavernous sinus and to consider it a partial one as the sinus lumen was not totally occluded. However, the important narrowing of the siphon and the fact that granulomatous tissue gathered around it forming a periarterial cuff, suggest that the lesion originated in the arterial wall.

Ecker (1945), Chavany, Guiot, and Poloukhine (1952), and others have repeatedly observed segmentary spasms of the carotid siphon in the angiograms of intracranial aneurysms. If, in addition, we bear in mind that for various reasons the aneurysmal sac may not be visible in the angiogram, as shown, among many others, by the cases of Alpers and Ryan (1949), it can easily be realized that angiography may not be sufficient to distinguish between a case like the one submitted here and a case of aneurysm of the parasellar region.

Local or segmentary arterial narrowing must not, therefore, always be interpreted as pathognomic of a thrombo-angeitic or endoarteritic process reducing the lumen of the vessel, but only as the effect of a lesion of the wall of the artery of the

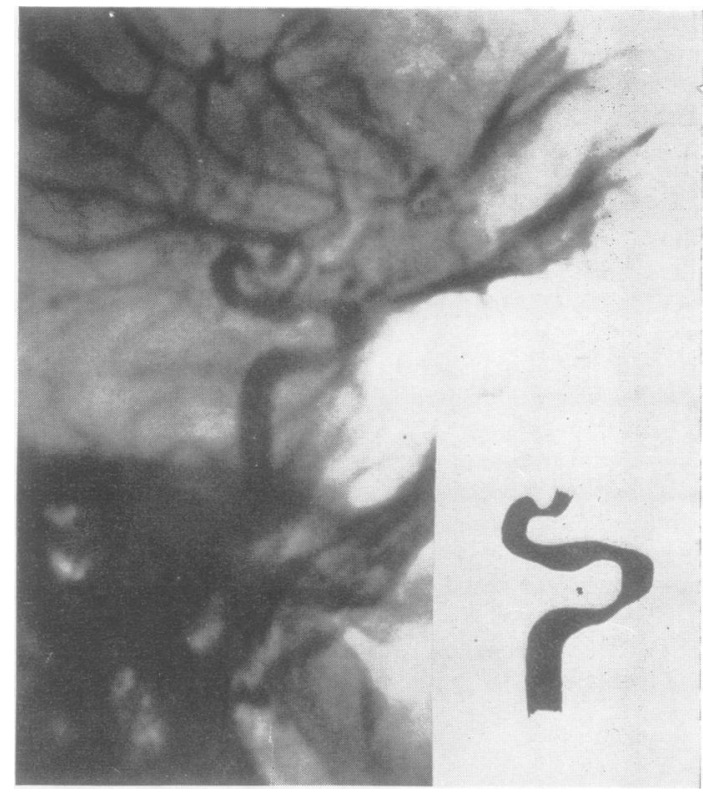

Fig. 3

most varied nature, or of merely a segmentary spasm. In this case, the calibre of the left carotid was obviously decreased in segment $C_{2}$, not by a partial obstruction of the lumen, but by the constriction of the vessel in the whole of its perimeter. The rigidity of the carotid walls shows very clearly that this narrowing was due to an organic process, whereas the arteriographic narrowing of $\mathrm{C}_{2}$ must be referred to spasm.

\section{Summary}

A case is submitted with a post-mortem report of a periarteritic process of the carotid siphon with the clinical picture of a carotid infraclinoidal aneurysm. The arteriographic examination showed only a segmentary narrowing of segment $\mathrm{C}_{2}$ of the carotid siphon which did not differ essentially from the local vascular spasms that may be seen in cases of intracranial aneurysms.

\section{REFERENCES}

Alpers, B. J., and Ryan, J. J. (1949). J. nerv. ment. Dis., 109, 220. Chavany, J. A., Guiot, G., and Poloukhine, P. (1952). Presse méd., Davis, L., 1803.

(1947). Brit. J. Surg., 34, 307.

Ecker, A. D. (1945). J. Neurosurg., 2, 479.

Kefferson, G. (1938). Brit. J. Surg., 26, 267.

List, D. F., and Hodges, F. J. (1946). J. Neurosurg., 3, 25. 